\title{
Role of the C-terminal region of dextransucrase from Leuconostoc mesenteroides IBT-PQ in cell anchoring
}

\author{
Clarita Olvera, José Luis Fernández-Vázquez, \\ Luis Ledezma-Candanoza and Agustín López-Munguía
}

\begin{abstract}
Correspondence
Agustín López-Munguía

agustin@ibt.unam.mx
\end{abstract}

Received 10 April 2007

Revised 28 August 2007

Accepted 13 September 2007 Departamento de Ingeniería Celular y Biocatálisis, Instituto de Biotecnología, Universidad Nacional
Autónoma de México (UNAM), Cuernavaca, Morelos, Mexico

\section{INTRODUCTION}

Glucose polymers and their corresponding oligosaccharides can be synthesized through enzymic transglucosylation by a wide variety of glycansucrases capable of using sucrose as substrate. Among this group, dextransucrases (EC 2.4.1.5) are glucansucrases (GSs) able to produce dextran, a glucose polymer linked mainly through $\alpha 1-6$ bonds, although $\alpha 1-3, \alpha 1-6, \alpha 1-4$ and $\alpha 1-2$ bonds are also found, in both the main chain and the branching linkages (Bozonnet et al., 2002; Kralj et al., 2002). Furthermore, glucose can also be transferred to molecules that can act as nucleophile acceptors; in the case of sugars such as maltose, a wide variety of low-molecular-mass glucose oligosaccharides are produced (Monsan \& Paul, 1995).

Several GSs have been the subject of intensive research. They are mainly produced by Streptococcus, Lactobacillus and Leuconostoc species, where they may be both constitutive and sucrose-induced, and are obtained either as soluble proteins in the culture medium or as cellassociated proteins (Hamada \& Slade, 1980; Kralj et al., 2002; Monsan et al., 2001).

On average, GS genes encode proteins of $\sim 1600$ aa, equivalent to a molecular mass of $160 \mathrm{kDa}$, and although no crystal structure is yet available, different functional domains have already been identified (van Hijum et al.,

Abbreviations: ChBR, choline binding region; CWBR, cell wall binding region; GBD, glucan binding domain; GS, glucansucrase; LTA, lipoteichoic acid.

The GenBank/EMBL/DDBJ accession number for the entire dsrP gene sequence of Leuconostoc mesenteroides IBT-PQ is AY504865.
2006). GSs belong to family 70 of glycoside hydrolases (http://www.cazy.org). A 36-40 aa signal peptide, characteristic of extracellular enzymes and bearing $50 \%$ identity among dextransucrases, has been found in the $\mathrm{N}$-terminal region. The signal peptide is followed by a variable region, which in some cases contains repeat sequences such as those known as A and TQQ repeats or $\mathrm{T}$ and $\mathrm{E}$ motifs (Kralj et al., 2004; Bozonnet et al., 2002). The catalytic domain contains $\sim 1000$ aa, including the three highly conserved carboxylic amino acids of the catalytic triad: two aspartic acid residues and one glutamic acid residue also present in $\alpha$-amylases (Monchois et al., 1999a; Matsuura et al., 1984). It has been shown that in Leuconostoc mesenteroides NRRL B-512F dextransucrase (DsrS), the aspartic acid in position 551 is essential for catalytic activity, while glutamic acid 589 and aspartic acid 662 complement the catalytic triad (Monchois et al., 1997). In fact, it has been proposed that the catalytic domain of the GSs from L. mesenteroides and Streptococcus species have the secondary structure of a circularly permuted $\alpha$ amylase type $(\beta / \alpha)_{8}$ barrel (MacGregor et al., 1996). Tsai et al. (2000) have predicted a TIM-barrel of $(\alpha / \beta)_{8}$ structure for the catalytic domain of Streptococcus mutans glucosyltransferase GtfB, based on the sequences and secondary structure alignment against the crystal structures reported for several $\alpha$-amylases.

Finally, the C-terminal end of GSs consists of a 400-500 aa region containing a series of related but non-identical tandem repeats of 20-30 aa each, divided into four classes according to their sequence, and designated as A, B, C and $\mathrm{D}$ repeats (Russell, 1990). This region is involved in binding to the glucan in streptococcal and lactobacilli GSs, 
and has therefore been designated the glucan binding domain (GBD) (Shah et al., 2004; Krajl et al., 2004a). However, there is controversy about its function in Leuconostoc dextransucrases, as although several authors report strong binding of the C-terminal region to dextran (Miller et al., 1986; Funane et al., 1998), other authors are not able to find this interaction (Shah et al., 2004).

The repeat units present in dextransucrase C-terminal regions resemble those found in the cell wall binding motif in the choline binding domain (ChBD) of autolysin from Streptococcus pneumoniae, as well as in other surfacelocated proteins from several bacteria (Janecek et al., 2000). These modules bind choline to the lipoteichoic acid (LTA) that serves to anchor the target proteins to the cell surface. The crystal structure of a ChBD from autolysin LytA of $S$. pneumoniae has been determined, revealing a solenoid fold consisting exclusively of $\beta$-hairpins that stack to form a left-handed superhelix. DsrB, a glucosyltransferase from $L$. mesenteroides NRRL B-1299 and GtfI GBD of Streptococcus downei are found among the proteins with high identity to the ChBD (Fernández-Tornero et al., 2001). Recently, the crystal structure of the 255-residue C terminal of TcdA from Clostridium difficile bound to a synthetic derivative of a natural carbohydrate receptor, $\alpha$-Gal- $(1,3)-\beta$-Gal- $(1,4)$ $\beta$ - GlcNAcO $\left(\mathrm{CH}_{2}\right) 8 \mathrm{CO}_{2} \mathrm{CH}_{3}$, was determined (Ho et al., 2005). This region also adopts a $\beta$-solenoid fold. Residues that interact with the carbohydrate are conserved in all seven putative binding sites in TcdA, suggesting a multivalent binding mode (Greco et al., 2006).

Several streptococcal GSs and Leuconostoc dextransucrases are present in both the pellet and the supernatant fractions of cultures when strains are grown in the presence of sucrose; it is usually assumed that insoluble GSs are the result of binding to high-molecular-weight dextran that pellets together the enzyme and the cells. However, recent studies concerning the structures involved in cell anchoring have questioned this general hypothesis. For instance, Zahnley \& Smith (2000) have shown that alternansucrase is bound to L. mesenteroides cells in the absence of polymer.

We have already reported the production and properties of a cell-associated dextransucrase produced by $L$. mesenteroides IBT-PQ, a strain isolated from pulque, a traditional pre-Hispanic alcoholic beverage (Chellapandian et al., 1998). In the present study, we report the molecular and functional characterization of the $d s r P$ gene that encodes this enzyme, as well as the role in cell wall anchorage of its C-terminal region. A C-terminal domain model was built based on the cell wall binding domain; to date, to the best of our knowledge, no model has been proposed for this region in dextransucrases.

\section{METHODS}

Bacterial strains, media and growth conditions. L. mesenteroides IBT-PQ and NRRL B-1299 bacterial strains were grown at $30{ }^{\circ} \mathrm{C}$ and 200 r.p.m., as previously reported (Chellapandian et al., 1998), with
$20 \mathrm{~g}^{-1}$ glucose or xylose instead of sucrose as carbon source, in order to avoid enzyme and polymer synthesis, as these interfere with DNA extraction. Escherichia coli $\mathrm{DH} 5 \alpha$, the host for cloning, was grown at $37{ }^{\circ} \mathrm{C}$ and 250 r.p.m. in Luria-Bertani (LB) medium, supplemented where appropriate with $50 \mu \mathrm{g}$ kanamicin $\mathrm{ml}^{-1}$ or $100 \mu \mathrm{g}$ ampicillin $\mathrm{ml}^{-1}$ in order to maintain the plasmid construction. Agar $(1.5 \%, \mathrm{w} / \mathrm{v})$ was added for growth in solid medium.

Cloning and expression of the $L$. mesenteroides IBT-PQ dextransucrase gene. Molecular biology methods were carried out according to Sambrook \& Russell (2001). Based on sequence homology between conserved regions located in the catalytic core of different GS genes of Gram-positive bacteria, degenerate primers PQ + $1\left[5^{\prime}\right.$-AATGAT(A/G)TTGATAATTCAAATCCA-3'] and PQ-1 [5' A(A/G)CATAACGATCA $(\mathrm{G} / \mathrm{T})(\mathrm{C} / \mathrm{T})$ AAAAGCATA-3'] were designed, and were used for PCR with chromosomal DNA of $L$. mesenteroides strain IBT-PQ as template, resulting in the isolation of a single fragment of $1505 \mathrm{bp}$. Sequence analysis confirmed its GS identity. In order to obtain the $3^{\prime}$ region of the gene, Southern hybridization of chromosomal DNA of L. mesenteroides IBT-PQ digested with ClaI, using the $1505 \mathrm{bp}$ amplified PCR fragment as probe, was carried out. Results revealed a hybridizing fragment of about $3200 \mathrm{bp}$. The chromosomal DNA of L. mesenteroides IBTPQ digested with ClaI was then ligated to yield a circular DNA. This sample was used as template for a PCR with diverging primers PQ-2 (5'-CATATTCAGCTTCTGTGGTTGGTG-3') and $\mathrm{PQ}+2$ (5'-GAGTTGGGGAATTACGAACTTCG-3'), yielding an amplification product of $\sim 3200 \mathrm{bp}$. Based also on the $1505 \mathrm{bp}$ sequence, primers PQ-3 (5'-AAGTTTAACAAGTAATACATCCC-3') and PQ $+3\left(5^{\prime}\right.$-GATAGTGATGTCTCACAAAGC-3') were designed in order to isolate the $5^{\prime}$ region by inverse PCR. In the same way, L. mesenteroides IBT-PQ genomic DNA was digested with KpnI and ligated, yielding circular DNA that was used as the template for PCR, obtaining an amplification product of $\sim 3500 \mathrm{bp}$. Finally, a 5909 bp fragment containing the whole gene was amplified with primers $\mathrm{PQ}+4\left(5^{\prime}\right.$-CTTGTTTCCAAAGCGCTAATC- $\left.3^{\prime}\right)$ and PQ-4 (5'-ACAGAACTTGACCCAGATGTG-3'). The $\mathrm{G}+\mathrm{C}$ content was found to be similar to that of other Leuconostoc genes. The sequence of the entire $d s r P$ gene was submitted to GenBank.

The $d s r P$-encoding gene and its C-terminal region (343 aa) were cloned in an $E$. coli expression vector under the control of the inducible promoter (araBAD). Using primers $\mathrm{PQ}+5$ (5'-AGAAATAGAAATGTAACAAGCGT- $\left.3^{\prime}\right)$ and PQ-5 (5'-GCTTTTAATCAGCTCTCCAGA- $3^{\prime}$ ), the $d s r P$ gene was amplified, while the C-terminal region was amplified using the primers GBDDsrPfw (5' -CGCGGATCCGGGATGACATATTATTCTACAAGTGG-3') and GBDDsrPrev (5'-CCCAAGCTTGCTTTTAATCAGCTCTCCAGAATTTGC-3'). Both fragments were cloned in the pBAD-TOPO Thiofusion vector (Invitrogen), which adds thioredoxin in the N-terminal and a Histag in the C-terminal regions, leading to plasmids pBAD- $d s r P$ and pBAD-dsrPCTR. The expression was carried out in E. coli TOP10, after optimization of arabinose concentration. The Thio-DsrS-His was produced using the construction and procedure reported by Moulis et al. (2006b), and was kindly provided by Professor Magali Remaud-Simeon, INSA-Toulouse, France.

Preparation of recombinant DsrP, DsrS and GBD-DsrP in E. coli. E. coli Top10 harbouring the aforementioned constructions was grown to $\mathrm{OD}_{600} 0.5$. The cells were induced with $0.02 \%(\mathrm{w} / \mathrm{v})$ arabinose, incubated for $4 \mathrm{~h}$ and harvested by centrifugation $(5000 \mathrm{~g}$ at $4{ }^{\circ} \mathrm{C}$ for $10 \mathrm{~min}$ ). The resulting pellet was washed twice with $50 \mathrm{mM}$ sodium acetate buffer, $\mathrm{pH}$ 5.2, and broken by ultrasonication with four $10 \mathrm{~s}$ pulses of $10 \mu \mathrm{m}$ with $3 \mathrm{~min}$ intervals. Cell debris was removed by centrifugation, and the cell extracts of DsrP and DsrS were assayed for activity. The initial rate of the dextransucrase reaction was measured at $30{ }^{\circ} \mathrm{C}$ in $50 \mathrm{mM}$ sodium acetate buffer, 
$\mathrm{pH} 5.2$, in the presence of $0.3 \mathrm{M}$ sucrose. The dextransucrase activity was measured by following the release of reducing sugar from sucrose by the 3,5-dinitrosalicylic acid (DNS) method. One activity unit (U) was defined as the amount of enzyme producing one micromole of fructose equivalent per minute. The amount of glucose produced by hydrolytic activity was found to be negligible as determined by HPLC. Denatured samples of the extracted proteins were electrophoretically separated on $6 \%$ polyacrylamide gels and stained with Coomassie Brilliant Blue. The proteins of the cellular extract were separated on SDS-PAGE gels, followed by pre-incubation in $50 \mathrm{mM}$ sodium acetate buffer, $\mathrm{pH}$ 6.5, and $1 \%$ Tween 80 . In situ polymer synthesis was then induced by overnight incubation in the same buffer supplemented with 0.3 M sucrose (Figures \& Edwards, 1979) in order to identify the enzymes. Two SDS-PAGE activity gels were carried out in parallel, and after incubation with sucrose, the buffer was changed to $50 \mathrm{mM}$ sodium acetate buffer, $\mathrm{pH} 6.0$, and one of the two gels was treated with dextranase (Sigma) at $37{ }^{\circ} \mathrm{C}$ for $12 \mathrm{~h}$, in order to identify those bands where dextran had been synthesized. GBD DsrP in cell extracts was analysed by Western blotting with anti-His antibodies. The Western blots were performed using the immunoblot detection method described by Coligan et al. (2007). Detection of C-terminal His-tags was carried out using antibodies from the QIAexpress antiHis HRP conjugate kit (Qiagen) and visualized with tetramethylbenzidine (TMB; Zymed).

Preparation of wild dextransucrases (DsrP and DsrE) and noninduced $L$. mesenteroides IBT-PQ cells for binding assays. The cell-associated wild enzymes from $L$. mesenteroides IBT-PQ and NRRL B-1299 were obtained from $500 \mathrm{ml}$ cultures in LM medium (Chellapandian et al., 1998) with sucrose as inducer. After $4-6$ h of culture, the cells were centrifuged and extensively washed with $50 \mathrm{mM}$ phosphate buffer, $\mathrm{pH}$ 6.5. The enzyme was then extracted by treatment of the pellet recovered in $12.5 \mathrm{ml} 8 \mathrm{M}$ urea at $25{ }^{\circ} \mathrm{C}$ for $1 \mathrm{~h}$ with occasional gentle shaking, as previously described (OlivaresIllana et al., 2003). After centrifugation, the extract was dialysed against $10 \mathrm{mM}$ phosphate buffer, $\mathrm{pH} 6.5$, and analysed by SDSPAGE. In order to prepare L. mesenteroides IBT-PQ cells devoid of the enzyme, cultures were prepared in LM broth supplemented with xylose as carbon source. After $8 \mathrm{~h}$ of growth, the cells were harvested and washed twice with $50 \mathrm{mM}$ phosphate buffer, $\mathrm{pH} 6.5$.

Binding assays. Binding assays were carried out in $5 \mathrm{ml}$ experiments by placing into contact $0.5 \mathrm{mg} \mathrm{ml}^{-1}$ of purified wild dextransucrase (DsrP or DsrE), recombinant DsrS or the His-tagged C-terminal region (not purified) with $2.5 \mathrm{mg} \mathrm{ml}^{-1}$ of cells at $4{ }^{\circ} \mathrm{C}$ with shaking for $12 \mathrm{~h}$. After incubation, the cells were pelleted by centrifugation and washed twice with $50 \mathrm{mM}$ phosphate buffer, $\mathrm{pH}$ 6.5. Cells and supernatants were then subjected to electrophoresis and assayed for dextransucrase activity. In the case of GBD DsrP, the His-tag Cterminal region was detected by Western blotting with anti-His antibodies.

Molecular modelling. The DsrP protein sequence was submitted to BLAST (http://www.ncbi.nlm.nih.gov/BLAST/), Geno3d (http:// geno3d-pbil.ibcp.fr) (Combet et al., 2002) and swiss-MODEL (http:// www.expasy.org/swissmod/SWISS-MODEL.html) (Schwede et al., 2003; Guex \& Peitsch, 1997; Peitsch, 1995) to search for structural homologues. The alignments and models retrieved were used to construct a molecular model of DsrP C-terminal domains. Computer procedures were performed on a Silicon Graphics IRIS Solid Impact workstation. Modelling, graphic display, energy minimization, molecular dynamics and analysis were carried out with the program INSIGHT II, and with the HOMOLOGY, BIOPOLYMER, DISCOVER and DOCKING packages (Accelrys). We used the default parameters for minimization (steepest, derivative 0.001, charges, CVFF force field) and molecular dynamics (equilibration 100, history 10, charges, temperature 300.00, time step 1.00, CVFF force field), except for the number of iterations for minimization and steps for dynamics, in which we used 200 and 20000 (20 psec), respectively. All minimizations and molecular dynamics were carried out with the molecule soaked with a $5 \AA$ water layer.

\section{RESULTS AND DISCUSSION}

\section{Molecular characterization of $L$. mesenteroides strain IBT-PQ dextransucrase gene}

Based on sequence homology between conserved regions located in the catalytic core of different GS genes of Grampositive bacteria, degenerate primers were designed and used in a PCR with chromosomal DNA of L. mesenteroides strain IBT-PQ as template. The resulting fragment was used as probe to obtain the $3^{\prime}$ region by Southern blotting and the $5^{\prime}$ region by inverse PCR, as described in Methods. One complete ORF was found in the compiled sequence of $4365 \mathrm{bp}$, and encoded a putative GS gene that we designated $d s r P$. A potential promoter sequence upstream from $\operatorname{ds} P$ (79 bp from the start codon) was identified, with a -35 sequence (CTGACA) separated by 23 bp from a -10 sequence (TATGAT). Also, an ATG start codon preceded by an 8 bp upstream putative RBS (AGGGAA) was observed. A search of the entire nucleotide sequence revealed one incomplete ORF situated upstream of $d s r P$ on the opposite strand. This ORF was preceded by a putative RBS (TGGGTC). From a BLAST search of the translated amino acid peptide sequence, an identity of $21 \%$ with the transcription regulator $\mathrm{rgg}$ from GtfD of Streptococcus gordonii was found (Vickerman et al., 2001).

The $d s r P$ gene, which encodes a protein of 1454 aa, with a deduced molecular mass of $160586 \mathrm{Da}$ and a pI of 4.5, was cloned and expressed. The highest dextransucrase activity was obtained when E. coli TOP10 (pBAD-dsrP) cells were incubated with $0.02 \%(\mathrm{w} / \mathrm{v})$ arabinose, added at $\mathrm{OD}_{600}$ 0.5. Under these conditions, after $4 \mathrm{~h}$, the activity reached $0.06 \mathrm{U}$ (mg protein $)^{-1}$. The polymers produced by recombinant $\mathrm{DsrP}$, the wild-type enzyme produced by $L$. mesenteroides IBT-PQ, and the industrial strain $L$. mesenteroides NRRL B-512F used as a control, were readily digested after incubation of SDS-PAGE activity gels with dextranase, as described in Methods, demonstrating that they were all dextrans.

Alignment of the amino acid sequence of DsrP with that of other GSs by BLAST revealed clear similarities. The putative protein structure of DsrP contains (i) an N-terminal signal sequence of 41 aa, (ii) a relatively large variable $\mathrm{N}$-terminal domain of 250 aa, (iii) a catalytic domain of 820 aa, and (iv) a C-terminal domain of 343 aa. The highest similarity ( $89 \%$ identity and $90 \%$ similarity) at the amino acid level was found with DsrT from L. mesenteroides NRRL B-512F. The gene $d s r T$ encodes a dextransucrase-like protein that is truncated after the catalytic domain by the deletion of five nucleotides. The $d s r T$ gene product does not synthesize dextran, but the insertion of the five nucleotides CAGAT in the $d s r T$ gene $(d s r T 5)$ and its expression in E. coli resulted 


\begin{tabular}{|c|c|c|c|}
\hline & 61 & D ATTT DKS & 68 \\
\hline & 71 & TNDËATTTĀDTS & 82 \\
\hline & 83 & TNDKATTTADTS & 94 \\
\hline & 95 & TNDKATTTADTS & 106 \\
\hline & 107 & TNDKATTTADIS & 118 \\
\hline & 119 & TNNKATTTADTS & 130 \\
\hline & 131 & TNNKAATTADTS & 142 \\
\hline sensus & & TNDKATTTADTS & \\
\hline
\end{tabular}

Fig. 1. N-terminal region repeat units of the GS DsrP. Bold type indicates conserved amino acids.

in a $210 \mathrm{kDa}$ enzyme (DsrT5) with dextransucrase activity (Funane et al., 2000).

The deduced N-terminal amino acid sequence of DsrP contains a putative secretion peptide with a predicted signal peptidase cleavage site between amino acids 40 and 41 (http://www.cbs.dtu.dk/services/SignalP/). Within the deduced N-terminal variable region of DsrP, a series of seven repeat units with a mean length of $12 \mathrm{aa}$, similar to motif $\mathrm{T}$ present in DsrT described by Bozonnet et al. (2002), was found (Fig. 1). The same kind of repeat units with a different consensus (motif $S$ ) were also identified by those authors in DsrE, while in GTF33, nine repeats of 14 aa were found and designated TTQ repeats by Krajl et al. (2004b). Repeat units with a different consensus have also been found in fructansucrases, such as inulosucrase IslA from Leuconostoc citreum (Olivares-Illana et al., 2003), designated motif I, in levansucrase LevC (motif C) from $L$. mesenteroides ATCC 8293 (Olvera et al., 2007), and in fructansucrase FtfA (motif F) from Lactobacillus sanfranciscensis (Tieking et al., 2005).

The putative catalytic domain of DsrP showed high similarity (about $50 \%$ identity and $60 \%$ similarity) to other known Streptococcus and Leuconostoc GS catalytic domains. In DsrP this domain is found between amino acids 292 and 1111, and contains the essential conserved amino acids required for enzymic activity. Analysis of DsrP sequences suggests that the amino acids of the catalytic triad correspond to aspartic acids 473 and 589, and glutamic acid 511.

The C terminal in DsrP is formed by 343 aa, starting from position 1112. Five 65 aa imperfect sequences were identified in this region (Fig. 2a). Within these sequences, five type A repeats were found, according to the consensus sequences proposed by Russell (1990), also shown in Fig. 2(a). However, an alternative description of this region is possible from the consensus with the choline binding region (ChBR) or cell wall binding region (CWBR) of LytA reported by Fernández-Tornero et al. (2001). A comparison between DsrP C-terminal repeats and LytA CWBR is shown in Fig. 2(b). In this case, five putative repeats were also identified. CWBR repeats have been reported in DsrE from L. mesenteroides NRRL B-1299 (Bozonnet et al.,

(a)

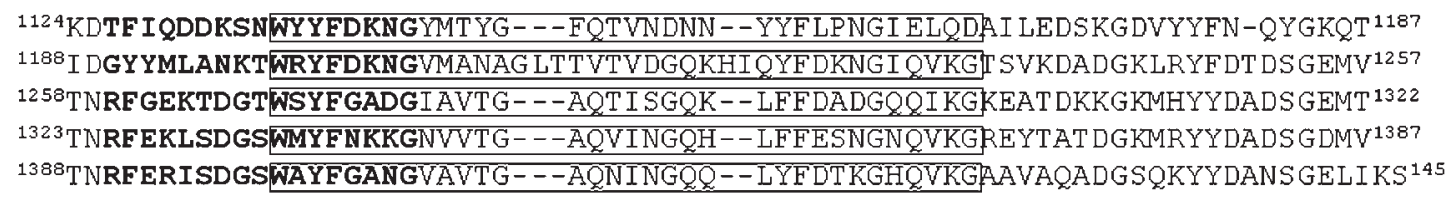

(b)

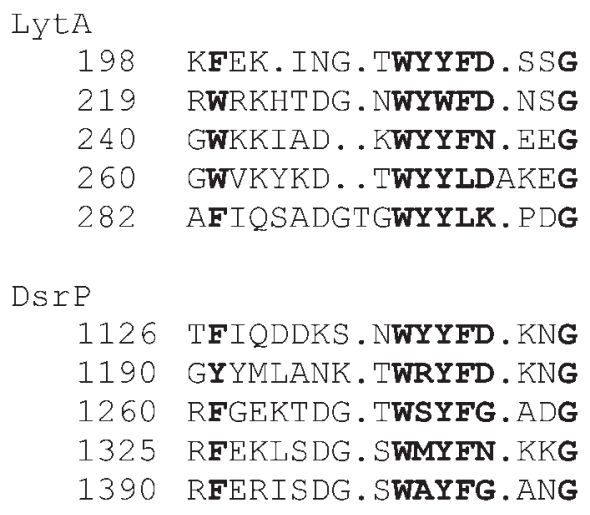

Fig. 2. (a) A repeats (in boxes) and putative ChBRs (in bold type) found within the 65 aa imperfect repeat sequences in the DsrP C-terminal region. (b) Comparison between repeat sequences of the DsrP C-terminal domain and those of LytA ChBR (consensus in bold type). 
2002), another cell-associated GS, in DsrB from $L$. mesenteroides and in GtfI from S. downei (FernándezTornero et al., 2001). Different functions have been suggested for the GS C-terminal domain, including roles in glucan polymerization, in glucan structure, in the transfer of products from the catalytic site, and in the cellsurface localization of GSs. However, the precise role of the C terminal remains unknown (Janecek et al., 2000; Kato \& Kuramitsu, 1991).

\section{DsrP C-terminal region molecular model}

A DsrP C-terminal domain model was constructed based on the known structures of the CWBR of LytA and ToxA (GenBank accession nos 1GVMB and 2F6EA) (FernándezTornero et al., 2001, 2002; Ho et al., 2005; Greco et al., 2006). Both the Geno3D and the swISs-MODEL programs retrieved equivalent structural models from C-terminal domain sequences. Although the DsrP C-terminal domain is longer than that of LytA CWBR, it was easy to model, as it may be described as a modular sequence of LytA CWBR motifs. All the different repeats were independently modelled and later joined into a single structure. This model was soaked with a $5 \AA$ layer of water, energyminimized and improved using molecular dynamics. This resulted in a DsrP C-terminal domain model with several similarities to LytA CWBR but also with an important overall difference. LytA CWBR is an asymmetric heterodimer linked through a hydrophobic patch in each CWBR repeat, forming a structure similar to a boomerang (Fernández-Tornero et al., 2001), while the DsrP Cterminal model is a continuous extended structure with a shape similar to a cigar, bent at its centre by $\sim 20^{\circ}$ (Fig. 3). As in LytA CWBR, there is a sequence regularity represented by the repeat units found in the DsrP C-terminal domain that fits well with the 'modular' character of the left-handed helix of CWBR in LytA. The DsrP C-terminal domain sequence has several imperfect repeats with a mean length of $\sim 65$ aa. These structural repeats are $\mathrm{K}^{1124}-\mathrm{T}^{1187}, \mathrm{I}^{1188}-\mathrm{T}^{1258}, \mathrm{~N}^{1259}-\mathrm{T}^{1323}, \mathrm{~N}^{1324}-$ $\mathrm{T}^{1388}$ and $\mathrm{N}^{1389}-\mathrm{S}^{1454}$ (Fig. 2a). Within these structural motifs, A repeats, usually present in these regions (Janecek et al., 2000; Shah et al., 2004), are found. Moreover, other repeat units found in this type of protein, such as IDGYYFD + N + G (von Eichel-Streiber et al., 1992) and the Y-G repeats (Giffard \& Jacques, 1994), are also contained in these structural motifs (Fig. 3). We also found several repeats in the DsrP sequence, with a YFDANG consensus, forming part of an equivalent $\beta$-hairpin structure in the model. All these repeats reflect the cyclic modular nature of the DsrP C-terminal model. Five regions similar to cell wall binding sites found in LytA CWBR were identified in the

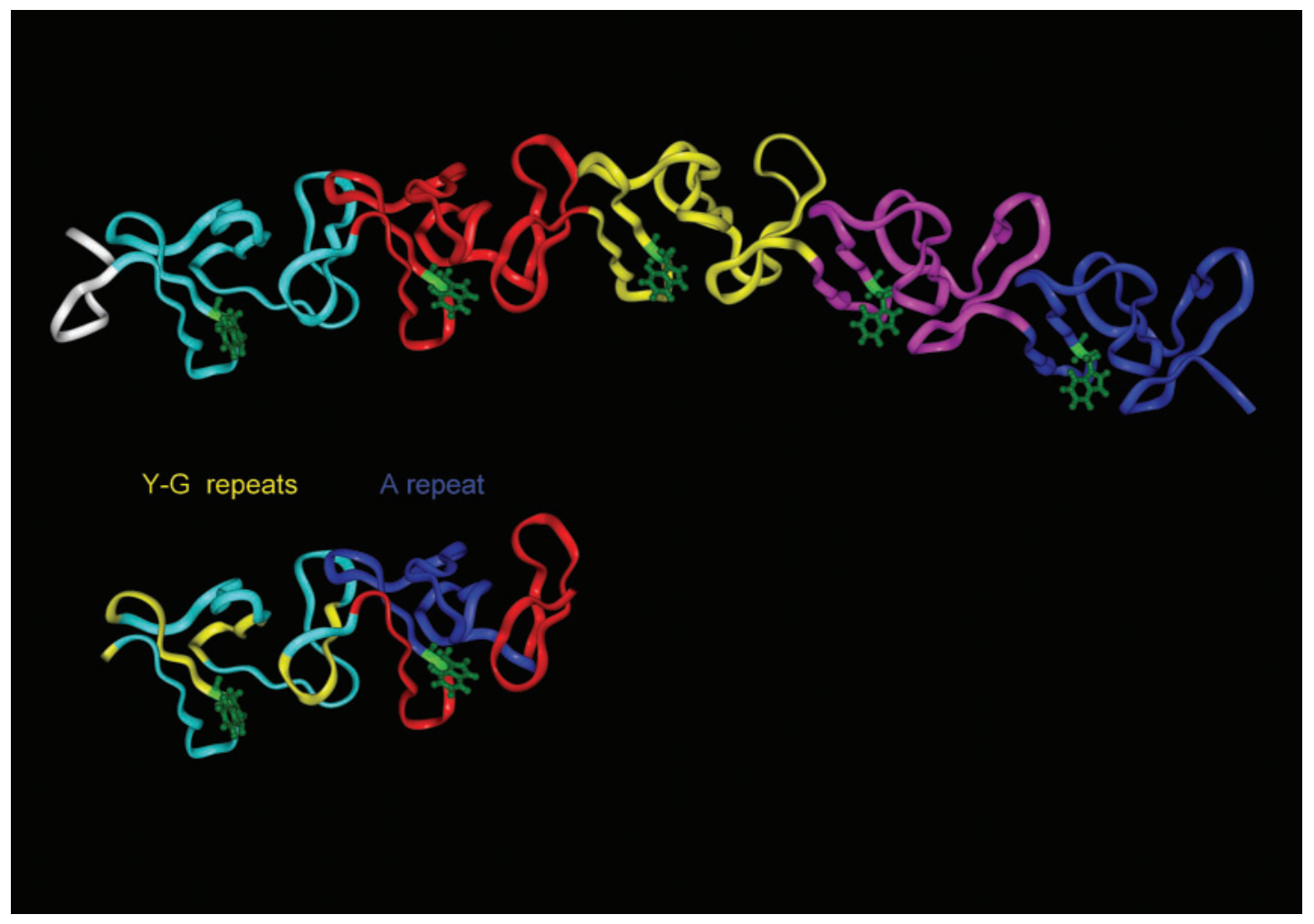

Fig. 3. Upper panel, ribbon representation of the DsrP C-terminal region consisting of five 65 aa imperfect sequences; lower panel, localization of the $Y-G$ and $A$ repeats within the $\mathrm{C}$-terminal repeats in the first two imperfect sequences. The figure was obtained with Insight II. 


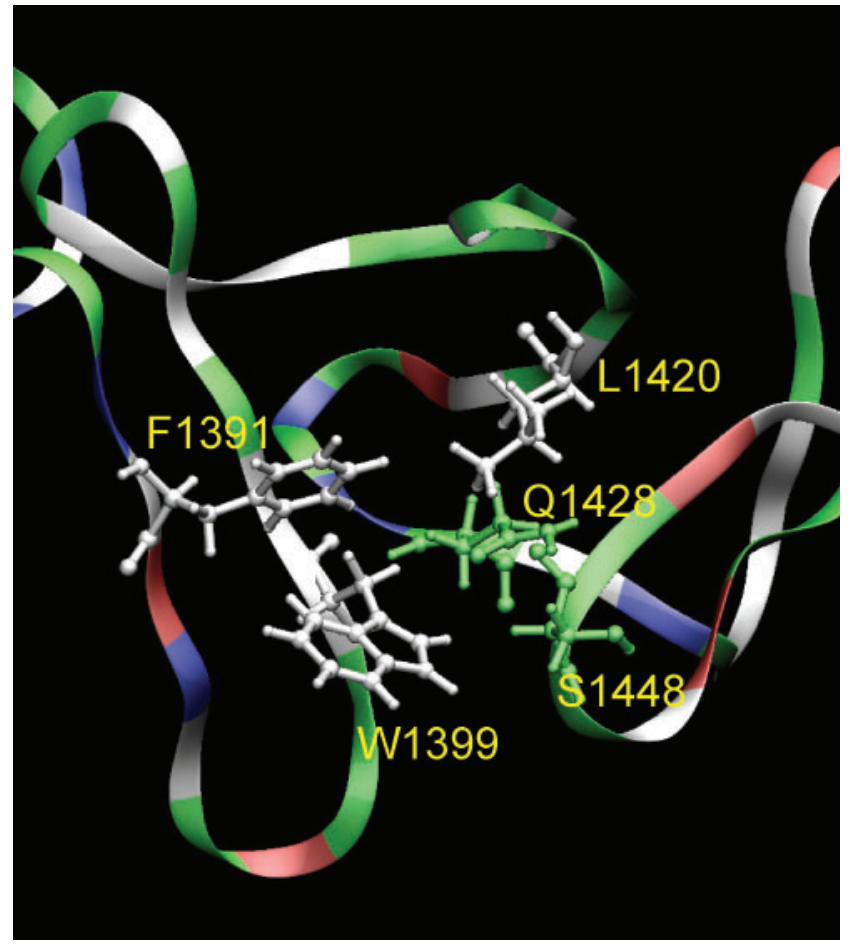

Fig. 4. Ribbon representation of the putative CWBR repeat binding pocket of DsrP, showing the residues probably involved in binding. The colour shows the type of amino acid residue: blue, basic; red, acidic; green, polar; white, non-polar. The figure was obtained with VMD.

DsrP C-terminal domain. In LytA, these sites serve to attach the protein through teichoic acids and LTAs to the cell wall of S. pneumoniae (Fernández-Tornero et al., 2001, 2002). Repeats similar to cell wall binding pockets are formed in our model by a combination of five residues, mainly hydrophobic. This is illustrated in Fig. 4 for the fifth site, which is formed by $\mathrm{F}^{1391}, \mathrm{~W}^{1399}, \mathrm{~L}^{1420}, \mathrm{Q}^{1428}$ and $\mathrm{S}^{1448}$. In the same way, the cell wall binding pockets in LytA are formed by a combination of hydrophobic residues $\left(\mathrm{F}^{199}\right.$, $\mathrm{W}^{206}, \mathrm{Y}^{229}, \mathrm{M}^{237}$ and $\mathrm{E}^{254}$ for the first CWBR repeat in LytA) (Fernández-Tornero et al., 2001).

Evidence in favour of our model prediction is provided by circular dichroism studies performed on the GBD region of GtfI from $S$. downeii, in which a high $\beta$-sheet and loop content without any proportion of $\alpha$-helix was found, in agreement with the LytA CWBR structure and with our model (Monchois et al., 1999a).

Although the functionality of these sites remains to be clarified (see below), the model suggests that the C-terminal DsrP binds to the cell wall in L. mesenteroides, as all five putative cell wall binding sites are oriented to the same face of DsrP (Fig. 3). The hydrophobic and hydrophilic patterns in the DsrP C-terminal model are very similar to those found in LytA (not shown). The protein has hydrophilic residues located in the cleft formed by the $\beta$-hairpins of the solenoid that join the glucan unit of the teichoic acid/LTA, as suggested by Fernández-Tornero et al. (2001). As glucan could also be linked to the hydrophilic residues in this region, this domain could therefore have the double function of joining DsrP to the L. mesenteroides cell wall and to the glucan matrix. In fact, this double function of the C-terminal domain in GSs has already been proposed for GtfI of S. mutans (Kato \& Kuramitsu, 1991).

\section{DsrP anchoring to the cell wall}

In order to explore the ability of DsrP to anchor the $L$. mesenteroides cell wall, cells devoid of DsrP were produced in xylose cultures to avoid induction. Afterwards, the non-induced cells were placed in contact with a partially purified preparation of DsrP. Other GSs were also included in this experiment: DsrE from L. mesenteroides NRRL B1299, which is also a cell-associated enzyme that contains CWBR repeats in the C-terminal domain; and DsrS from $L$. mesenteroides NRRL B-512F as a negative control, on the basis that it lacks CW repeats. After intensive washing it was found that both DsrP and DsrE were retained and active on the surface of $L$. mesenteroides cells, as shown in Fig. 5(a, b), while DsrS was not retained (Fig. 5c). This was demonstrated basically by enzyme activity assays of the cells and the enzyme solution, before and after contact among them. These results suggest that DsrE and DsrP CWBR repeat units are involved in cell attachment. As DsrE and DsrP also contain repeat units in the N-terminal region ( $\mathrm{E}$ and $\mathrm{T}$ motifs, respectively), binding could also result from attachment mediated by interactions through this region. In fact, in a similar experiment, LevC, a levansucrase from $L$. mesenteroides ATCC 8293 that has repeats (C motif) in the $\mathrm{N}$ terminus, did not attach to the cell wall.

In LytA, the CWBR repeats recognize choline in the LTA that serves to anchor the protein to S. pneumoniae cells. In order to determine whether the CWBR repeats in DsrP have a similar function, the binding experiments described above were performed in the presence of choline, phosphorylcholine and PAF-18 (an antagonist of choline). Contrary to what was expected, the binding of DsrP to the cell wall was not inhibited in the presence of choline or the two analogous compounds. Similarly, DsrP was not retained by DEAEcellulose, an affinity support commonly used for the purification of choline-binding proteins. These results suggest that the attachment of DsrP to the L. mesenteroides cell wall is not mediated by the LTA choline moieties. In fact, the cell envelope of $L$. mesenteroides contains only traces of choline $(<2 \%)$, phosphatidyl glycerol $(50 \%)$ being the major component of LTA phospholipids.

\section{The DsrP $C$ terminal is involved in anchoring to the $L$. mesenteroides cell wall}

In order to demonstrate that DsrP is bound to the $L$. mesenteroides cell wall through the $\mathrm{C}$-terminal domain, a 
(a) DsrP

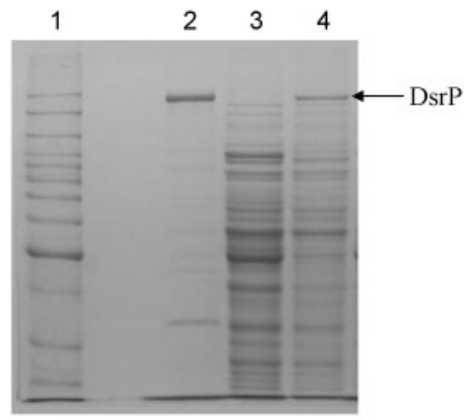

(c) DsrS

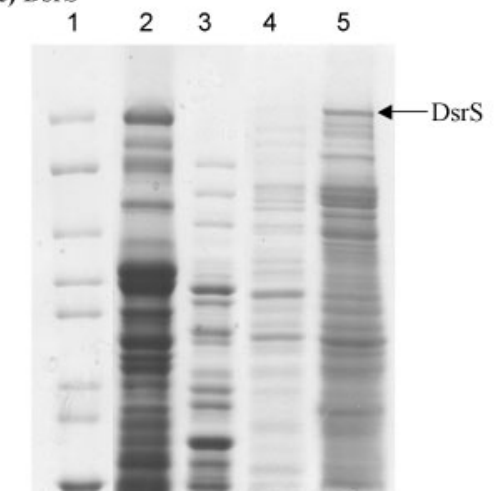

(b) DsrE

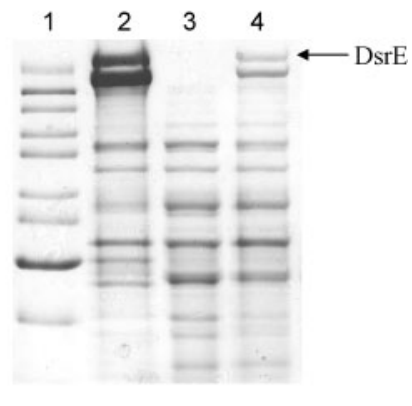

Fig. 5. Binding assays of dextransucrases with (a) DsrP, (b) DsrE and (c) DsrS. Lanes: 1, molecular weight marker; 2 , partially purified dextransucrase; 3, non-induced $L$. mesenteroides cells; 4, washed non-induced cells after contact with dextransucrase; 5 , supernatant after contact with dextransucrase.

\section{$\begin{array}{lllllll}1 & 2 & 3 & 4 & 5 & 6 & 7\end{array}$}

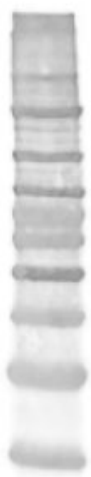

Fig. 6. Western blot analyses of the His-tagged DsrP C-terminal fragment placed in contact with non-induced $L$. mesenteroides IBT-PQ cells. Lanes: 1, molecular weight marker; 2, non-induced L. mesenteroides IBT-PQ cells; 3, His-tagged DsrP C-terminal fragment in solution; 4, His-tagged DsrP C-terminal fragment in solution after contact with non-induced cells; 5 , non-induced $L$. mesenteroides IBT-PQ cells after contact with the His-tagged DsrP C-terminal fragment; 6, His-tagged DsrP C-terminal fragment in solution after contact with non-induced cells in the presence of $10 \%$ Dextran T10; 7, non-induced L. mesenteroides IBT-PQ cells after contact with His-tagged DsrP C-terminal fragment cells in the presence of $10 \%$ Dextran T10.
His-tagged DsrP C-terminal fragment of 343 aa $(38 \mathrm{kDa})$ was expressed in E. coli. Contact experiments similar to those performed with the whole protein were carried out with the single C-terminal region of DsrP. As shown in Fig. 6, the attachment of the DsrP C-terminal domain to the noninduced L. mesenteroides IBT-PQ cells was detected by Western blot analysis using anti-His antibodies, demonstrating that this domain binds to the cell wall. This is probably the function of the C-terminal domain in other cellassociated GSs, such as alternansucrase, DsrE (which in a previous experiment was demonstrated to bind L. mesenteroides IBT-PQ cells) and GtfB from S. mutans, which contains CWBR repeats in its C-terminal domain.

It has been suggested that dextran associates with the A repeats in dextransucrase: Funane et al. (1998) isolated four peptides bound to glucan from L. mesenteroides NRRL B-512F dextransucrase, one of these peptides having an A repeat sequence. Based on the reaction products of truncated versions of DsrS, Moulis et al. (2006a) have suggested that A repeats interact with dextran during polymer synthesis, and possibly support the anchoring of the growing polymer to the enzyme surface. Considering this additional function of the C-terminal domain, an experiment was performed to find out if cell-anchoring and dextran binding properties are mutually exclusive. Cellcontact assays of the His-tagged DsrP C-terminal region were therefore performed in the presence of $10 \%$ Dextran $\mathrm{T} 10$, in order to determine whether dextran inhibits cell 
wall anchoring. It was found that in spite of the dextran, the C-terminal region retained the ability to anchor to the cell surface, demonstrating that the protein cell wall binding sites do not interact with dextran, as suggested in the structural model. This may be the consequence of different sites for glucan and cell wall binding. Further experiments are required to demonstrate the nature of the interactions among DsrP, glucan and the cell wall.

\section{ACKNOWLEDGEMENTS}

This project was supported by Consejo Nacional de Ciencia y Tecnología (CONACyT) no. 40609-Z and by UNAM (PAPIIT no. IN238202). We thank Dr. X. Soberon for the support with modelling infrastructure. We also thank Fernando Gonzalez, Sara G. Centeno Leija and Ma. Elena Rodriguez for their technical support, and Eugenio López, Santiago Becerra, Paul Gaytán and Jorge Yañez for the primer synthesis and DNA sequences.

\section{REFERENCES}

Bozonnet, S., Dols-Laffargue, M., Fabre, E., Pizzut, S., RemaudSimeon, M., Monsan, P. \& Willemont, R. (2002). Molecular characterization of DSR-E, an $\alpha-1,2$ linkage-synthesizing dextransucrase with two catalytic domains. J Bacteriol 184, 5753-5761.

Chellapandian, M., Larios, C., Sanchez-Gonzalez, M. \& LopezMunguia, A. (1998). Production and properties of a dextransucrase from Leuconostoc mesenteroides IBT-PQ isolated from "pulque", a traditional Aztec alcoholic beverage. J Ind Microbiol Biotechnol 21, $51-56$.

Coligan, J. E., Duna, B. M., Speicher, D. W. \& Wingfield, P. T. (2007). Current Protocols in Protein Science. Bognor Regis, UK: John Wiley and Sons.

Combet, C., Jambon, M., Deleage, G. \& Geourjon, C. (2002). Geno3D: automatic comparative molecular modelling of protein. Bioinformatics 18, 213-214.

Fernández-Tornero, C., López, R., García, E., Giménez-Gallego, G. \& Romero, A. (2001). A novel solenoid fold in the cell wall anchoring domain of the pneumococcal virulence factor LytA. Nat Struct Biol 8, 1020-1024.

Fernández-Tornero, C., García, E., López, R., Giménez-Gallego, G. \& Romero, A. (2002). Two new crystal forms of the choline-binding domain of the major pneumococcal autolysin: insights into the dynamics of the active homodimer. J Mol Biol 321, 163-173.

Figures, W. R. \& Edwards, J. R. (1979). Resolution of the glycosyltransferase activities from two strains of Streptococcus mutans by polyacrylamide gel electrophoresis in the presence of Tween 80 . Biochim Biophys Acta 577, 142-146.

Funane, K., Ookura, T. \& Kobayashi, M. (1998). Glucan binding regions of dextransucrase from Leuconostoc mesenteroides NRRL B512F. Biosci Biotechnol Biochem 62, 123-127.

Funane, K., Mizuno, K., Takahara, H. \& Kobayashi, M. (2000). Gene encoding a dextransucrase-like protein in Leuconostoc mesenteroides NRRL B-512F. Biosci Biotechnol Biochem 64, 29-38.

Giffard, P. M. \& Jacques, N. A. (1994). Definition of a fundamental repeating unit in streptococcal glucosyltransferase glucan-binding regions and related sequences. J Dent Res 73, 1133-1141.

Greco, A., Ho, J. G., Lin, S. J., Palcic, M. M., Rupnik, M. \& Ng, K. K. (2006). Carbohydrate recognition by Clostridium difficile toxin A. Nat Struct Mol Biol 13, 460-461.
Guex, N. \& Peitsch, M. C. (1997). SWISS-MODEL and the Swiss PdbViewer: an environment for comparative protein modelling. Electrophoresis 18, 2714-2723.

Hamada, S. \& Slade, H. D. (1980). Biology, immunology, and cariogenicity of Streptococcus mutans. Microbiol Rev 44, 331-384.

Ho, J. G. S., Greco, A., Rupnik, M. \& Ng, K. K.-S. (2005). Crystal structure of receptor-binding repeats from Clostridium difficile toxin. Proc Natl Acad Sci U S A 102, 18373-18378.

Janecek, S., Svensson, B. \& Russell, R. (2000). Location of repeat elements in glucansucrases of Leuconostoc and Streptococcus species. FEMS Microbiol Lett 192, 53-57.

Kato, C. \& Kuramitsu, H. K. (1991). Molecular basis for the association of glucosyltransferases with the cell surface of oral streptococci. FEMS Microbiol Lett 63, 153-157.

Kralj, S., van Geel-Schutten, G. H., Rahaoui, H., Leer, R. J., Faber, E. J., van der Maarel, M. J. \& Dijkhuizen, L. (2002). Molecular characterization of a novel glucosyltransferase from Lactobacillus reuteri strain 121 synthesizing a unique, highly branched glucan with $\alpha-(1 \rightarrow 4)$ and $\alpha-(1 \rightarrow 6)$ glucosidic bonds. Appl Environ Microbiol 68, $4283-4291$

Kralj, S., van Geel-Schutten, G. H., van der Maarel, M. J. \& Dijkhuizen, L. (2004a). Biochemical and molecular characterization of Lactobacillus reuteri 121 reuteransucrase. Microbiology 150, 2099-2112.

Kralj, S., Van Geel-Schutten, G. H., Dondorff, M. M. G., Kirsanovs, S., Van Der Maarel, M. J. E. C. \& Dijkhuizen, L. (2004b). Glucan synthesis in the genus Lactobacillus: isolation and characterization of glucansucrase genes, enzymes and glucan products from six different strains. Microbiology 150, 3681-3690.

MacGregor, E. A., Jesperen, H. M. \& Svensson, B. (1996). A circularly permuted $\alpha$-amylase-type $\alpha / \beta$-barrel structure in glucansynthesizing glucosyltransferases. FEBS Lett 378, 263-266.

Matsuura, Y., Kusunoki, M., Harada, W. \& Kakudo, M. (1984). Structure and possible catalytic residues of Taka-amylase A. J Biochem (Tokyo) 95, 697-702.

Miller, A. W., Eklund, S. H. \& Robyt, J. F. (1986). Milligram to gram scale purification and characterization of dextransucrase from Leuconostoc mesenteroides NRRL B-512F. Carbohydr Res 147, 119-133.

Monchois, V., Remaud-Simeon, M., Russell, R., Monsan, P. \& Willemont, R. (1997). Characterization of Leuconostoc mesenteroides NRRL B-512F dextransucrase (DSR-S) and identification of aminoacid residues playing a key role in enzyme activity. Appl Microbiol Biotechnol 48, 465-472.

Monchois, V., Lakey, J. H. \& Russell, R. R. (1999a). Secondary structure of Streptococcus downei GTF-1 glucansucrase. FEMS Microbiol Lett 177, 243-248.

Monchois, V., Willemont, R. \& Monsan, P. (1999b). Glucansucrases: mechanism of action and structure-function relationships. FEMS Microbiol Rev 23, 131-151.

Monsan, P. \& Paul, F. (1995). Enzymatic synthesis of oligosaccharides. FEMS Microbiol Rev 16, 187-192.

Monsan, P., Bozonnet, S., Albenne, C., Joucla, G., Willemont, R.-M. \& Remaud-Simeon, M. (2001). Homopolysaccharides from lactic acid bacteria. Int Dairy J 11, 675-685.

Moulis, C., Joucla, G., Harrison, D., Fabre, E., Potocki-Veronese, G., Monsan, P. \& Remaud-Simeon, M. (2006a). Understanding the polymerization mechanism of glycoside-hydrolase family 70 glucansucrases. J Biol Chem 281, 31254-31267.

Moulis, C., Arcache, A., Escalier, P. C., Rinaudo, M., Monsan, P., Remaud-Simeon, M. \& Potocki-Veronese, G. (2006b). High-level production and purification of a fully active recombinant dextransu- 
crase from Leuconostoc mesenteroides NRRL B-512F. FEMS Microbiol Lett 261, 203-210.

Olivares-Illana, V., López-Munguía, A. \& Olvera, C. (2003). Molecular characterization of inulosucrase from Leuconostoc citreum: a fructosyltransferase within a glucosyltransferase. J Bacteriol 185, 3606-3612.

Olvera, C., Centeno-Leija, S. \& Lopez-Munguia, A. (2007). Structural and functional features of fructansucrases present in Leuconostoc mesenteroides ATCC 8293. Antonie Van Leeuwenhoek 92, 11-20.

Peitsch, M. C. (1995). Protein modeling by E-mail. Biol/Technology 13, 658-660.

Russell, R. R. (1990). Molecular genetics of glucan metabolism in oral streptococci. Arch Oral Biol 35, 53S-58S.

Sambrook, J. \& Russell, D. W. (2001). Molecular Cloning: a Laboratory Manual, 3rd edn. Cold Spring Harbor, NY: Cold Spring Harbor Laboratory.

Schwede, T., Kopp, J., Guex, N. \& Peitsch, M. C. (2003). SWISSMODEL: an automated protein homology-modeling server. Nucleic Acids Res 31, 3381-3385.

Shah, D. S., Joucla, G., Remaud-Simeon, M. \& Russell, R. R. (2004). Conserved repeat motifs and glucan binding by glucansucrases of oral streptococci and Leuconostoc mesenteroides. J Bacteriol 186, 8301-8308.

Tieking, M., Ehrmann, M. A., Vogel, R. F. \& Ganzle, M. G. (2005). Molecular and functional characterization of a levansucrase from the sourdough isolate Lactobacillus sanfranciscensis TMW 1.392. Appl Microbiol Biotechnol 66, 655-663.

Tsai, Y. W., Chia, J. S., Shiau, Y. Y., Chou, H. C., Liaw, Y. C. \& Lou, K. L. (2000). Three-dimensional modelling of the catalytic domain of Streptococcus mutans glucosyltransferase GtfB. FEMS Microbiol Lett 188, 75-79.

van Hijum, S. A. F. T., Kralj, S., Ozimek, L. K., Dijkhuizen, L. \& van Geel-Schutten, I. G. H. (2006). Structure-function relationships of glucansucrase and fructansucrase enzymes from lactic acid bacteria. Microbiol Mol Biol Rev 70, 157-176.

Vickerman, M. M., Minick, P. E. \& Mather, N. M. (2001). Characterization of the Streptococcus gordonii chromosomal region immediately downstream of the glucosyltransferase gene. Microbiology 147, 3061-3070.

von Eichel-Streiber, C., Saueborn, M. \& Kuramitsu, H. K. (1992). Evidence for a modular structure of the homologous repetitive $\mathrm{C}$ terminal carbohydrate-binding sites of Clostridium difficile toxins and Streptococcus mutans glucosyltransferases. J Bacteriol 174

Zahnley, J. C. \& Smith, M. R. (2000). Cellular association of glucosyltransferases in Leuconostoc mesenteroides and effects of detergent on cell association. Appl Biochem Biotechnol 87, 57-70.

Edited by: D. A. Mills 